\title{
How Useful is Computed Tomography of the Chest for the Diagnosis of Asymptomatic Right Ventricular Perforation Following Pacemaker Insertion?
}

\author{
Maryam Ayati, Syamkumar Divakaramenon Menon, Carlos A Morillo, Jeffrey Healey and Stuart J Connolly
}

Arrhythmia and Pacing Service, Hamilton Health Sciences, McMaster University, Hamilton, Canada

DOI: http://doi.org/10.17925/EJAE.2016.02.01.42

\begin{abstract}
Insertion of implantable cardiac devices such as pacemaker and defibrillators is one of the most common invasive procedures in cardiology. This procedure can be sometimes very challenging and needs more advanced modalities to diagnose the complication. In this article, we present a case of asymptomatic right ventricular perforation post pacemaker implantation who was diagnosed with CT scan and was managed further.

\section{Keywords}

Pacemaker implantation, computed tomography scan, right ventricular rupture

Disclosure: Maryam Ayati, Syamkumar Divakara Menon, Carlos A Morillo, Jeffrey Healey and Stuart J Connolly have nothing to declare in relation to this article. No funding was received for the publication of this article.

Open Access: This article is published under the Creative Commons Attribution Noncommercial License, which permits any noncommercial use, distribution, adaptation, and reproduction provided the original author(s) and source are given appropriate credit.

Compliance with Ethics: All procedures were followed in accordance with the responsible committee on human experimentation and with the Helsinki Declaration of 1975 and subsequent revisions, and informed consent was received from the patient involved in this case study.

Received: 18 February 2016 Accepted: 3 March 2016 Citation: European Journal of Arrhythmia \& Electrophysiology, 2016;2(1):42-3

Correspondence: Maryam Ayati, Hamilton Health Sciences, McMaster University, Hamilton Canada. E: Aiaty50@gmail.com
\end{abstract}

Pacemaker (PM) insertion is one of the most common invasive procedures in cardiology. This routine procedure may be associated with unfortunate complications such as myocardial perforation. Although this is a welldescribed but rare complication in symptomatic patients, subclinical perforation is even rarer and the diagnosis remains a challenge. In subclinical and late manifestation, it is even more difficult to diagnose. ${ }^{1}$

Conventional imaging modalities such as echocardiography and chest X-ray are helpful for diagnosis but may be limited by several factors. We present a case of myocardial perforation post-PM insertion with the lead migrating to the lateral part of the seventh rib, in which conventional imaging modalities such as 2D-echo or chest X-ray were unable to clarify the diagnosis.

\section{Case presentation}

A 94-year-old lady referred for recurrent syncope complicated by T10 spinal fracture and documented transient complete heart block with a 13 second pause while on holter monitoring, underwent single chamber pacemaker insertion (Medtronic active fixation 5776-58 cm lead). Two weeks later, she was seen for routine follow-up at our device clinic. The device interrogation revealed no sensing or pacing despite programming to the highest sensitivity and output and 4,000 Ohms for impedance. The patient was in sinus rhythm and experienced no recurrent syncope or any further cardiac symptoms.

Chest X-ray (Figure 1) was unable to determine whether the tip of the lead was extracardiac. 2D-echo did not add any additional information and ruled out pericardial effusion due to poor window in an obese

\section{Figure 1: Chest X-ray}

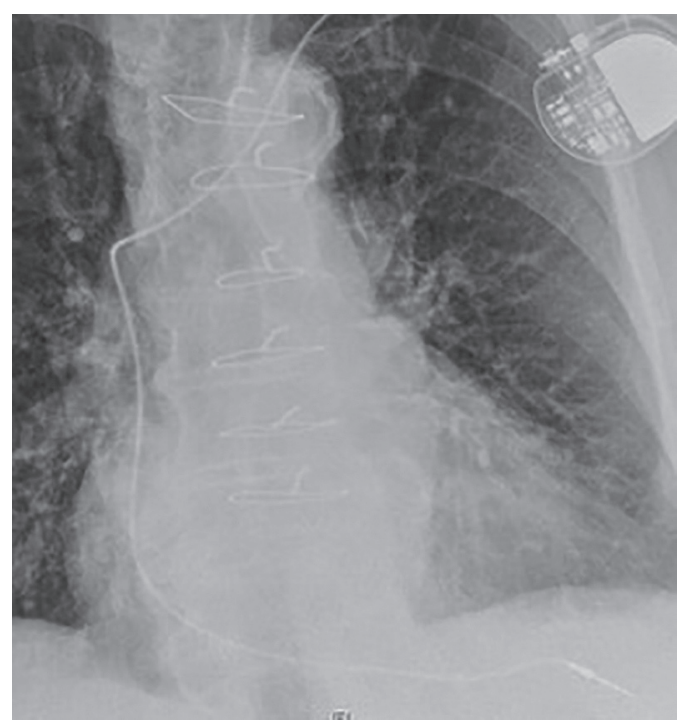

patient. A chest computed tomography (CT) scan showed the tip of the PM had perforated the right ventricle, extended $5.3 \mathrm{~cm}$ from epicardial fat and terminated along the costochondral junction of left seventh rib. This could be seen even better with the help of 3D pictures (Figures 2 and 3). The patient underwent an uncomplicated lead removal and a new passive ventricular lead insertion was performed with an $\mathrm{R}$ wave of $15.5 \mathrm{mV}$ and threshold of $0.25 \mathrm{mV}$, and resistance of $921 \mathrm{Ohms}$. The patient was followed for three months post-operatively, with no changes 


\section{Figure 2: Computed tomography scan}

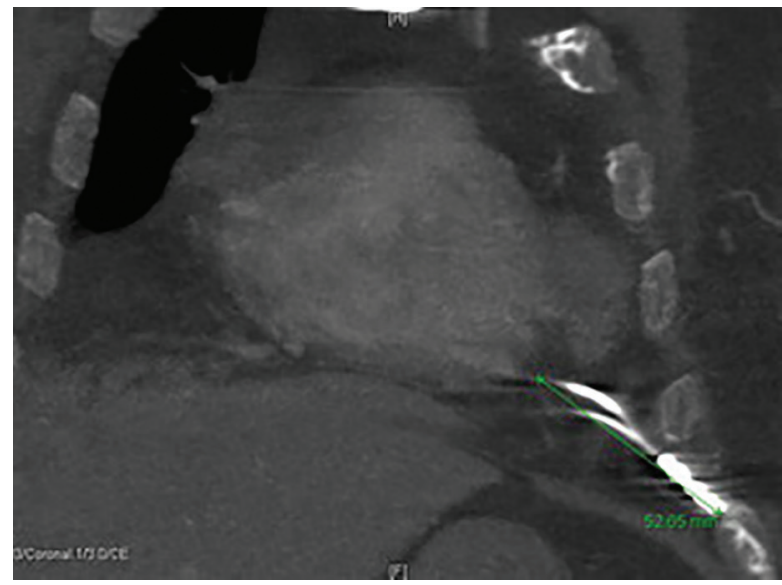

in measurements documented. This case highlights the usefulness of chest CT scan for the diagnosis of asymptomatic and subclinical ventricular perforation post-PM insertion in a patient presenting at the device clinic, in which lack of pacing at maximum output, loss of sensing and severely increased lead impedance were documented. Interestingly, this case did not show any pericardial effusion, as this is commonly seen with ventricular rupture. ${ }^{2}$ Even after manipulation and pulling back the PM lead, no pericardial tamponade occurred. ${ }^{3}$

\section{Discussion}

Although right ventricular (RV) perforation due to PM leads insertion is a rare complication, it could be potentially life threatening. Previously, it was thought that this happens more with active fixation leads,

\section{Figure 3: Computed tomography scan}

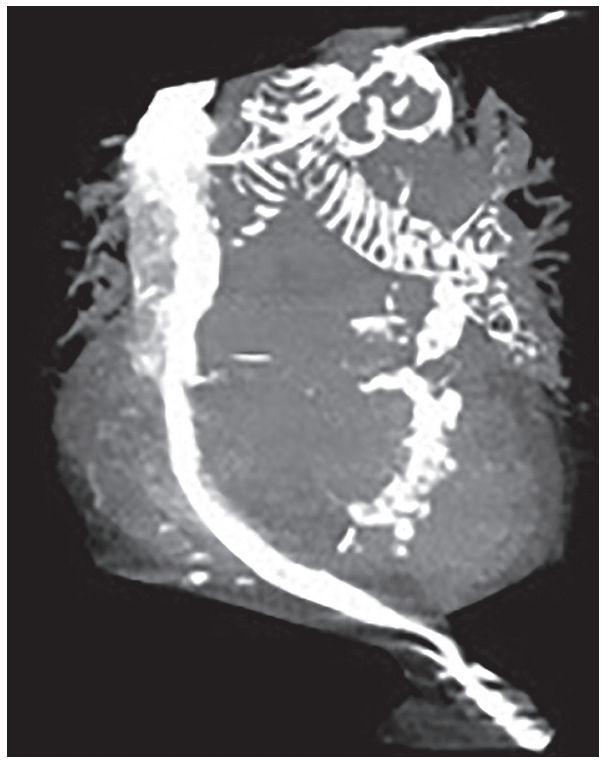

however, in one recent study published by Migliore et al. ${ }^{4}$ this was not proved. This study showed no statistically significant difference in RV perforation between active versus passive fixation leads ( 0.5 versus $0.3 \% ; p=0.3)$. In addition, there was no significant difference in RV perforation between PM and defibrillator leads.

Our case was done with an active fixation lead and was asymptomatic until PM data measurement post-implant. To explant and reposition the leads, we recommend having a cardiac surgeon on standby.

\footnotetext{
Guardado J, Pereira VH, Fernandes M, et al., Late right ventricular perforation: a rare complication of a pacemaker implantation, Rev Port Cir Cardiotorac Vasc, 2012;19:17-9.

Howell C, Bergin JD, A case report of pacemaker lead perforation causing late pericardial effusion and subacute cardiac tamponade, I Cardiovasc Nurs, 2005;20:271-5.

Khan MF, Zubairi ABS, Cardiac tamponade after removal of temporary pace maker in multidiscipinary intensive care unit, J Pak Med ASSOC, 2008;58:270-2.

Migliore F, Zorzi A, Bertaglia E, et al., Incidence, management, and prevention of right ventricular perforation by pacemaker and implantable cardioverter defibrillator leads, Pacing Clin Electrophysiol,
} 2014;37:1602-9. 